\title{
Terapia nutricional parenteral em CTI de hospital universitário
}

\author{
Parenteral nutrition therapy in the CTI of a university \\ hospital
}

Terapia nutricional parenteral en la CTI de un hospital universitario

Thaís Otranto Dias ${ }^{1}$ Fernanda Zanoni Consolo ${ }^{2}$

${ }^{1}$ Nutricionista pela Faculdade de Ciências Farmacêuticas, Alimentos e Nutrição (Facfan) da Universidade Federal de Mato Grosso do Sul (UFMS). E-mail: thais.otranto@gmail.com, Orcid: http://orcid.org/0000-0002-8516-0139

${ }^{2}$ Doutora e mestre pelo Programa de Pós-Graduação em Saúde e Desenvolvimento na Região Centro-Oeste, Universidade Federal de Mato

Grosso do Sul (UFMS). Docente do Curso de Nutrição e do Programa de Residência Multiprofissional em Atenção ao Paciente Crítico. E-mail: fernandazanoni@yahoo.com.br, Orcid: http://orcid.org/0000-0002-0812-2817 
Resumo: A Nutrição Parenteral (NP) é uma alternativa de suporte nutricional, podendo contribuir para recuperação da saúde e maior chance de sobrevida dos pacientes. Objetivou-se avaliar a adequação calórica e de macronutrientes da NP fornecida a pacientes internados em um Centro de Terapia Intensiva (CTI) adulto. Trata-se de estudo transversal, com coleta de dados secundários dos prontuários dos pacientes. Foram analisados dados de 21 indivíduos, com predominância do sexo masculino e média de idade de 62,05 \pm 14,59 anos. Quando consideradas as médias e os percentuais de adequação, apenas os lipídios mostraram-se inadequados, revelando aporte lipídico insuficiente em 52,6\% dos pacientes. Contudo a avaliação das prescrições indicou aporte calórico excessivo em 19,0\% da população, deficit proteico em 19,0\% e excesso de proteínas em 38,0\% dos casos. Conclui-se que existem inconformidades que impedem suporte nutricional apropriado, fato que ressalta a necessidade de monitoramento e reavaliação constante dos pacientes e da conduta nutricional adotada.

Palavras-chave: estado nutricional; nutrição parenteral; terapia nutricional; Centro de Terapia Intensiva.

\begin{abstract}
Parenteral Nutrition (PN) is an alternative of nutritional support, which contributes to patients' recovery and a greater chance of survival. The study aims to evaluate the adequacy of $\mathrm{PN}$ in terms of calories and macronutrients in patients admitted to an adult Intensive Care Unit (CTI, in the Portuguese acronym). This is a cross-sectional study, with secondary data collection from medical records. Data from 21 individuals were analyzed, with predominance of males and mean age of $62.05 \pm 14.59$ years. Considering the mean values and percentages of adequacy, only lipids were found to be inadequate, with insufficient lipid content in $52.6 \%$ of the patients. However, evaluation of PN prescriptions indicated excessive caloric intake in $19.0 \%$ of the population, protein deficit in $19.0 \%$, and protein excess in $38.0 \%$ of cases. Thus, some nonconformities prevent adequate nutritional support, which highlights the need for patients' monitoring and reassessment of nutritional management.
\end{abstract}

Keywords: nutritional status; parenteral nutrition; nutrition therapy; Intensive Care Unit.

Resumen: La Nutrición Parenteral (NP) es una alternativa de soporte nutricional que contribuye a la recuperación de la salud y supervivencia de los pacientes. Se objetivó evaluar la adecuación calórica y de macronutrientes de la NP suministrada a pacientes internados en una Unidad de Terapia Intensiva (CTI, en portugués) adulto. Es un estudio transversal con recolección de datos secundarios de los expedientes médicos. Se analizaron datos de 21 individuos, con predominio del sexo masculino y promedio de edad de 62,05 $\pm 14,59$ años. Considerando los promedios y porcentajes de adecuación, sólo los lípidos se mostraron inadecuados, con aporte insuficiente en el 52,6\% de los pacientes. La evaluación de prescripciones de NP indicó una ingesta calórica excesiva en el 19,0\% de la población, déficit proteico en 19,0\% y exceso de proteína en 38,0\%. Existen inconformidades que impiden el soporte nutricional apropiado, hecho que resalta la necesidad de monitorización constante de los pacientes y de la conducta nutricional.

Palabras clave: estado nutricional; nutrición parenteral; terapia nutricional; Unidad de Terapia Intensiva. 


\section{INTRODUÇÃO}

São considerados pacientes críticos aqueles com doença grave, iminente risco de morte ou perda de funcionalidade de órgão ou sistema do corpo humano e que necessitam de assistência médica imediata e constante. Geralmente, esses pacientes são internados em Centros de Terapia Intensiva (CTIs), a fim de provê-los com o suporte clínico necessário à sua recuperação.

O estado de saúde desses pacientes envolve disfunção de órgãos, hipermetabolismo, propensão a infecções, inflamação sistêmica, entre outros fatores que podem acarretar longo tempo de internação e altas taxas de mortalidade. Além disso, é frequente a identificação de desnutrição nessa população, o que pode contribuir para declínio do estado clínico, piora na qualidade de vida e maior morbimortalidade.

Devido a isso, a manutenção ou recuperação do estado nutricional de pacientes críticos é parte fundamental do tratamento e inclui diversos procedimentos terapêuticos, entre eles, o uso de nutrição enteral, parenteral, ou mesmo a associação entre essas vias. Por ser a mais fisiológica, a nutrição enteral é recomendada como primeira opção de escolha; contudo há contraindicações de seu uso em casos de obstrução intestinal, síndrome do intestino curto, isquemia mesentérica, entre outros agravos. Nessas circunstâncias, a Nutrição Parenteral (NP) pode viabilizar o suprimento das necessidades nutricionais dos pacientes. Apesar de seu uso permitir aporte nutricional adequado, requer monitoramento constante devido a intercorrências frequentemente associadas, como a hiperglicemia, distúrbios eletrolíticos e potenciais infecções.

Quando devidamente utilizado, o suporte nutricional parenteral auxilia na recuperação da saúde do paciente, proporcionando melhor evolução da doença e maior chance de sobrevida. Em virtude disso, é fundamental a avaliação dos resultados desse tratamento. Frequentemente, observa-se inadequação das prescrições de NP, subestimando ou superestimando as necessidades nutricionais. A gravidade do quadro clínico, ausência de protocolos clínicos para cálculo das necessidades nutricionais, interrupção frequente do uso da NP e/ou seu uso concomitante com outra via são alguns dos fatores que podem resultar no indevido aporte nutricional fornecido a esses pacientes. 
Diante dessa realidade, o presente estudo tem como objetivo avaliar a adequação calórica e de macronutrientes (carboidrato, proteína e lipídio) da NP fornecida a pacientes críticos internados em CTI adulto de um hospital universitário de Campo Grande, MS.

\section{MÉTODOS}

O estudo caracterizou-se como transversal, a partir da coleta de dados secundários de prontuários dos pacientes atendidos no CTI adulto do Hospital Universitário Maria Aparecida Pedrossian, da Universidade Federal de Mato Grosso do Sul (UFMS), no município de Campo Grande, MS.

A população investigada incluiu todos os pacientes internados no CTI entre abril de 2017 e abril de 2018 que receberam NP durante a internação. Foram excluídos do estudo os dados de seis pacientes: dois pacientes que não tinham seus arquivos disponíveis durante o período de coleta de dados; e quatro pacientes cujos dados de avaliação nutricional não constavam nos prontuários.

As informações coletadas dos prontuários incluíram dados para caracterização da população (sexo, data de nascimento, diagnóstico clínico), dados antropométricos (peso, altura e circunferência braquial), tipo e quantidade de suporte nutricional recebido, além de desfecho clínico dos pacientes.

A composição corporal foi avaliada por meio dos dados antropométricos, sendo que o Índice de Massa Corporal (IMC) foi obtido a partir do peso e estatura registrados nos prontuários, classificado segundo os pontos de corte da Organização Mundial da Saúde para indivíduos entre 20 e 59 anos (ORGANIZAÇÃO MUNDIAL DA SAÚDE [OMS], 1995) e, segundo Lipschitz (1994), para aqueles com idade igual ou superior a 60 anos. A Circunferência Braquial (CB) foi classificada conforme Blackburn e Thornton (1979).

As necessidades energéticas e de macronutrientes foram calculadas segundo cálculo simplificado com a oferta em $\mathrm{kcal} / \mathrm{kg} / \mathrm{dia}$ e gramas $/ \mathrm{kg} / \mathrm{dia}$ proposto pela European Society for Parenteral and Enteral Nutrition (Espen) para pacientes críticos. Tal recomendação propõe $25 \mathrm{kcal} / \mathrm{kg}$ de peso atual/ dia, de 1,3 grama a 1,5 grama de proteína/kg de peso atual/dia, $\geq 2$ gramas de carboidrato/kg de peso atual/dia, e de 0,7 grama a 1,5 grama de lipídios/ kg de peso atual/dia (SINGER et al., 2009). 
Para pacientes críticos obesos (IMC maior ou igual a $30 \mathrm{~kg} / \mathrm{m}^{2}$ ), utilizaram-se as recomendações energéticas e proteicas propostas pela American Society of Parenteral and Enteral Nutrition (Aspen), sendo elas de $11 \mathrm{kcal} / \mathrm{kg}$ a $14 \mathrm{kcal} / \mathrm{kg}$ de peso atual/dia para indivíduos com IMC entre $30 \mathrm{~kg} / \mathrm{m}^{2}$ e $50 \mathrm{~kg} / \mathrm{m}^{2} ; 22 \mathrm{kcal} / \mathrm{kg}$ a $25 \mathrm{kcal} / \mathrm{kg}$ de peso ideal/dia para aqueles com IMC superior a $50 \mathrm{~kg} / \mathrm{m}^{2} ; 2$ gramas de proteína/kg de peso ideal/ dia para pacientes com IMC entre $30 \mathrm{~kg} / \mathrm{m}^{2}$ e $40 \mathrm{~kg} / \mathrm{m}^{2}$; e 2 gramas a 2,5 gramas de proteína/kg de peso ideal/dia para aqueles com IMC superior a $40 \mathrm{~kg} / \mathrm{m}^{2}$ (MCCLAVE et al., 2016).

A análise da adequação do suporte nutricional baseou-se na prescrição correspondente ao dia em que o paciente recebeu NP plena, isto é, 100\% do planejado inicialmente. Em casos nos quais a evolução da NP não atingiu $100 \%$, utilizou-se a prescrição do dia correspondente ao maior aporte nutricional recebido por essa via.

Por ser uma complicação recorrente do uso de NP, verificou-se a presença de desvios na glicemia (hipoglicemia ou hiperglicemia) por meio dos valores do exame de glicemia registrados no prontuário referentes ao período em que o paciente utilizou o suporte parenteral. A avaliação se deu segundo os parâmetros estabelecidos pela American Diabetes Association (ADA, 2017) para pacientes hospitalizados, que considera hipoglicemia os valores inferiores a $70 \mathrm{mg} / \mathrm{dL}$, e hiperglicemia quando o resultado do exame é $\geq 180 \mathrm{mg} / \mathrm{dL}$.

Os dados foram digitados e tabulados em planilha do programa Microsoft Excel 2013. Para análise de dados, foi utilizada estatística descritiva por meio do cálculo de frequências absolutas, relativas, médias e desvios-padrão.

Este estudo foi submetido ao Comitê de Ética e Pesquisa Envolvendo Seres Humanos da Universidade Federal de Mato Grosso do Sul (UFMS), obtendo recebimento e aprovação para realização, sob o processo de n. 2.355.570.

\section{RESULTADOS}

O presente estudo utilizou dados de 21 pacientes, dos quais 76,2\% eram do sexo masculino; a média de idade em ambos os sexos foi de 62,0 
\pm 14,6 (23-81) anos. O tempo médio de internação no CTI foi de 19,9 \pm 13,3 (1-56) dias, sendo os principais diagnósticos clínicos as doenças que acometem o trato digestivo e respiratório, correspondendo a 47,6\% e 23,8\% dos casos, respectivamente (Tabela 1 ). A mortalidade no grupo avaliado correspondeu a $85,7 \%$ para ambos os sexos $(87,5 \%$ entre o sexo masculino e $80,0 \%$ entre o sexo feminino).

Tabela 1 - Diagnóstico clínico dos pacientes internados no CTI de acordo com localização da patologia. Hospital Universitário Maria Aparecida Pedrossian, Campo Grande, MS, 2017-2018 ( $n=21)$

\begin{tabular}{lccc}
\hline \multirow{2}{*}{ Diagnóstico clínico } & Feminino & Masculino & Total \\
\cline { 2 - 4 } & $\mathbf{n}(\mathbf{\%})$ & $\mathbf{n}(\%)$ & $\mathbf{n}(\%)$ \\
\hline Doenças do trato digestivo & $2(40,0)$ & $8(50,0)$ & $10(47,6)$ \\
Doenças respiratórias & $2(40,0)$ & $3(18,8)$ & $5(23,8)$ \\
Neoplasias & $1(20,0)$ & $1(6,2)$ & $2(9,5)$ \\
Hepatopatia alcoólica & - & $1(6,2)$ & $1(4,8)$ \\
Síndrome de Fournier & - & $1(6,2)$ & $1(4,8)$ \\
Síndrome de Wernicke & - & $1(6,2)$ & $1(4,8)$ \\
Pancreatite aguda & - & $1(6,2)$ & $1(4,8)$ \\
\hline
\end{tabular}

Fonte: Autoria própria.

Com relação ao estado nutricional, identificou-se a maior parte da população estudada como eutrófica, segundo o IMC (42,9\%), e 33,3\% dos indivíduos apresentaram baixo peso (Tabela 2). A avaliação de acordo com o sexo permitiu observar excesso de peso, especialmente entre as mulheres (40,0\%), quando comparadas aos homens. Quando considerada a CB, a soma dos pacientes com desnutrição de qualquer grau totalizou 61,2\%, representando deficit nutricional importante no grupo avaliado.

Quanto ao suporte nutricional, a média de uso de NP foi de 13,8 \pm 10,5 (1-44) dias. A maior parte dos pacientes (61,9\%) recebeu associação entre nutrição parenteral e enteral, 33,3\% utilizaram NP exclusiva e 4,8\% receberam NP associada à nutrição enteral e oral. Cabe ressaltar que, em 93,3\% dos casos em que houve uso de nutrição enteral, esta foi utilizada como dieta de trânsito. 
Tabela 2 - Frequência relativa do perfil antropométrico de pacientes internados no CTI, segundo o sexo. Hospital Universitário Maria Aparecida Pedrossian, Campo Grande, MS, 2017-2018 ( $n=21$ )

\begin{tabular}{lccc}
\hline \multirow{2}{*}{ Índices antropométricos } & Feminino & Masculino & Total \\
\cline { 2 - 4 } & $\mathbf{n ~ ( \% )}$ & $\mathbf{n}(\mathbf{\%})$ & $\mathbf{n}(\mathbf{\%})$ \\
\hline IMC & $2(40,0)$ & $5(31,3)$ & $7(33,3)$ \\
Baixo peso & $1(20,0)$ & $8(50,0)$ & $9(42,9)$ \\
Eutrofia & $2(40,0)$ & $1(6,3)$ & $3(14,3)$ \\
Sobrepeso & - & $2(12,5)$ & $2(9,5)$ \\
Obesidade & - & & $3(16,7)$ \\
\%CB (n= 5; 13) & $1(20,0)$ & $2(15,4)$ & $3(16,7)$ \\
Desnutrição grave & $2(40,0)$ & $3(23,1)$ & $5(27,8)$ \\
Desnutrição moderada & $1(20,0)$ & $5(38,5)$ & $6(33,3)$ \\
Desnutrição leve & $1(20,0)$ & - & $1(5,5)$ \\
Eutrofia & - & - & - \\
Sobrepeso & & $3(23,1)$ & \\
Obesidade & & & \\
\hline
\end{tabular}

Fonte: Autoria própria.

A distensão abdominal e a presença associada de distensão abdominal e débito na sonda destinada à nutrição enteral foram identificadas como os principais motivos de indicação para uso de suporte nutricional parenteral, ocorrendo em $41,2 \%$ e $23,5 \%$ dos casos, respectivamente. Outros motivos de indicação corresponderam a débito na sonda enteral (11,8\%), estase gástrica (11,8\%), hemorragia hepática e laceração do estômago (5,9\%) e dieta precoce pós-gastrectomia (5,9\%).

A avaliação do aporte energético dos pacientes constatou que a média do valor calórico prescrito foi de $24,39 \pm 5,29$ calorias/kg de peso, com adequação de 103,87 $\pm 26,12 \%$. A adequação glicídica recebida pelos pacientes foi de $98,68 \pm 5,74 \%$ quando consideradas as recomendações da Espen para pacientes críticos, enquanto a proteica foi de 106,35 $\pm 21,89 \%$, e a lipídica totalizou 85,56 \pm 18,96\% (Tabela 3).

Em 19,0\% dos casos ( $n=4$ ), o aporte calórico recebido excedeu as necessidades energéticas, atingindo adequação calórica superior a 140\%. Tratando-se dos macronutrientes, a oferta de carboidratos mostrou-se 
adequada em $94,7 \%$ dos pacientes. Apenas um paciente recebeu dieta hipocalórica (56,4\% de adequação) e com adequação glicídica de 75\%, cabendo ressaltar que ele foi a óbito antes da evolução completa da NP.

Tabela 3 - Adequação calórica e de macronutrientes dos pacientes internados na UTI. Hospital Universitário Maria Aparecida Pedrossian, Campo Grande, MS, 2017-2018 ( $n=21$ )

\begin{tabular}{ccc}
\hline Composição nutricional & Média & 士 DP \\
\hline Valor calórico prescrito $(\mathrm{kcal} / \mathrm{kg})$ & 24,39 & 5,29 \\
Adequação calórica $(\%)$ & 103,87 & 26,12 \\
Valor glicídico prescrito $(\mathrm{g} / \mathrm{kg})$ & 3,07 & 0,84 \\
Adequação glicídica $(\%)$ & 98,68 & 5,74 \\
Valor proteico prescrito $(\mathrm{g} / \mathrm{kg})$ & 1,59 & 0,34 \\
Adequação proteica $(\%)$ & 106,35 & 21,89 \\
Valor lipídico prescrito $(\mathrm{g} / \mathrm{kg})$ & 0,67 & 0,23 \\
Adequação lipídica $(\%)$ & 85,56 & 18,96 \\
\hline
\end{tabular}

Nota: DP: desvio-padrão.

Fonte: Autoria própria.

Das 21 prescrições de NP analisadas com relação ao fornecimento de proteínas, identificaram-se quatro casos $(19,0 \%)$ com deficit proteico, cujas adequações corresponderam a 65,0\%,69,2\%,69,2\% e 89,9\% das necessidades dos pacientes. Destes, dois não alcançaram NP plena durante a internação, o que justifica aporte inferior ao recomendado, porém os outros pacientes evoluíram integralmente, conforme prescrição. Tais indivíduos foram classificados como obesos segundo o IMC (ambos na faixa entre 30 $\mathrm{kg} / \mathrm{m}^{2}$ e $40 \mathrm{~kg} / \mathrm{m}^{2}$ ) e receberam 1,6 g e 1,9 g proteína/kg de peso ideal/dia. Ademais, identificou-se infusão proteica acima do recomendado em 38,0\% da amostra, com percentuais de adequação acima de $110 \%$.

Quando avaliada a adequação lipídica, 52,6\% da população de estudo recebeu aporte inferior ao recomendado. O mesmo paciente que apresentou inadequação calórica e glicídica importante destacou-se quanto ao aporte lipídico, pois o volume infundido em relação à necessidade totalizou apenas $28,6 \%$ de adequação, fato associado ao óbito precedente ao alcance de NP plena. 
Entre as possíveis complicações do suporte nutricional parenteral, destaca-se a instabilidade hemodinâmica, a qual foi identificada em 47,6\% da amostra, com maior prevalência entre indivíduos do sexo feminino $(60,0 \%)$ do que entre os do sexo masculino $(43,8 \%)$ (Tabela 4). Outra complicação frequente são as alterações glicêmicas, sendo a hiperglicemia a de maior ocorrência em ambos os sexos, presente em $45,0 \%$ dos pacientes.

A NP foi evoluída gradativamente em $90,5 \%$ dos casos, e a maior parte dos pacientes $(76,2 \%)$ utilizaram NP plena durante a internação, ou seja, atingiram $100 \%$ das metas calóricas e de macronutrientes estabelecidas pela prescrição. O recebimento de NP plena foi mais frequente entre os pacientes do sexo masculino $(81,2 \%)$ do que os do sexo feminino $(60,0 \%)$.

Tabela 4 - Frequência relativa da ocorrência de instabilidade hemodinâmica e distúrbios glicêmicos entre pacientes internados no CTI, segundo o sexo. Hospital Universitário Maria Aparecida Pedrossian, Campo Grande, MS, 2017-2018 ( $n=21)$

\begin{tabular}{lccc}
\hline \multirow{2}{*}{ Complicação } & Feminino & Masculino & Total \\
\cline { 2 - 4 } & $\mathbf{n}(\%)$ & $\mathbf{n}(\%)$ & $\mathbf{n}(\%)$ \\
\hline Instabilidade hemodinâmica & & & \\
$\quad$ Sim & $3(60,0)$ & $7(43,8)$ & $10(47,6)$ \\
$\quad$ Não & $2(40,0)$ & $9(56,2)$ & $11(52,4)$ \\
Distúrbios glicêmicos $(\mathbf{n}=4 ; 16)$ & & & \\
$\quad$ Hipoglicemia & $1(25,0)$ & $1(6,2)$ & $2(10,0)$ \\
$\quad$ Normoglicemia & $1(25,0)$ & $3(18,8)$ & $4(20,0)$ \\
$\quad$ Hiperglicemia & $1(25,0)$ & $8(50,0)$ & $9(45,0)$ \\
$\quad$ Hipoglicemia + hiperglicemia & $1(25,0)$ & $4(25,0)$ & $5(25,0)$ \\
\hline
\end{tabular}

Fonte: Autoria própria.

\section{DISCUSSÃO}

A análise do perfil epidemiológico dos pacientes permitiu identificar predominância do sexo masculino e de pacientes idosos. Tal achado é consistente com outros estudos, como o de Santos e Viana (2016), em que $58,5 \%$ da população internada em um CTI de hospital universitário pertencia ao sexo masculino e a idade média encontrada pelos autores foi de 
$51,7 \pm 18,6$ anos. Albuquerque, Silva e Souza (2017) também identificaram maior presença de homens internados $(53,0 \%)$ na área de terapia intensiva em um hospital de atenção terciária no estado do Rio de Janeiro, tendo como média de idade 66,5 \pm 19,4 anos, ligeiramente acima do constatado no presente estudo, mas ressaltando a presença de população idosa nos CTIs. A prevalência de homens nessas unidades pode estar associada ao menor uso dos serviços primários e secundários de saúde por esse grupo, enquanto a presença majoritária de idosos parece ter relação com o crescimento da população idosa no país e com a maior ocorrência de doenças que acompanha o aumento da idade (EL-FAKHOURI et al., 2016).

O tempo médio de internação (19,9 $\pm 13,3$ dias) foi superior ao identificado por outros estudos, como o de Roque, Tonini e Melo (2016), que identificou média de 8,9 $\pm 11,7$ dias. O tempo de permanência se relaciona com as características dos pacientes e do hospital, sendo que tempos mais longos podem indicar necessidade de melhora no atendimento prestado, já que esse padrão pode ser ocasionado por complicações relacionadas ao cuidado deficiente ou pela presença de eventos adversos durante a internação, inerentes à patologia do paciente (EL-FAKHOURI et al., 2016).

Evidentemente, as doenças do trato digestivo, quer sejam infecciosas, como diarreia, quer sejam vasculares, como isquemia mesentérica, foram as mais predominantes como diagnósticos clínicos em que o paciente pode necessitar suporte nutricional parenteral. As doenças respiratórias, a exemplo da pneumonia, também representaram grande parte dos diagnósticos encontrados nos pacientes de CTI que utilizaram NP. Em CTI no estado do Rio de Janeiro, observou-se ocorrência de doenças neurológicas como principal diagnóstico entre os indivíduos (19,0\%), sendo as doenças do aparelho respiratório classificadas como segunda condição clínica mais frequente (17,0\%) (ALBUQUERQUE; SILVA; SOUZA, 2017). Outro estudo, realizado com pacientes internados em CTI de um hospital público em Florianópolis, identificou as doenças do aparelho circulatório e as lesões físicas, envenenamento e outras causas externas como principais diagnósticos clínicos, correspondendo a 23,3\% e 21,3\% das internações, respectivamente (RODRIGUEZ et al., 2016). Em tal estudo, as doenças respiratórias foram mencionadas como quarta maior causa de internação, totalizando 9,2\% 
dos casos. Esse cenário permite-nos inferir que há uma variação entre os diagnósticos mais frequentes em pacientes críticos, mas que doenças do trato respiratório representam uma importante parcela dos pacientes internados em diferentes unidades de terapia intensiva.

Observou-se, por meio deste estudo, alta mortalidade, correspondendo a $85,7 \%$ dos pacientes em geral. Esse expressivo valor foi superior à mortalidade de 24,32\% encontrada por El-Fakhouri et al. (2016) e à de $37,74 \%$ identificada por Freitas, Nogueira e Hessel (2015), sendo que ambos os trabalhos também foram realizados com pacientes críticos. O Hospital Universitário Maria Aparecida Pedrossian é referência em média e alta complexidade, isto é, os pacientes são encaminhados pela rede de atenção básica ou após atendimento ambulatorial e clínico no próprio hospital; dessa forma, supõe-se que os pacientes sejam admitidos no hospital com doenças em estágios mais avançados e/ou com presença de complicações associadas, o que poderia culminar em pior desfecho clínico e contribuir para a alta mortalidade encontrada.

A avaliação do estado nutricional permitiu identificar que a maior parte da população estudada (42,9\%) apresentou eutrofia segundo o IMC. No entanto há uma grande proporção de indivíduos com baixo peso $(33,3 \%)$ e alta prevalência de sobrepeso, especialmente entre as mulheres (40\%). Outros estudos, como de Santos e Viana (2016) e Robinson et al. (2015), corroboram os resultados encontrados, em que se destaca a eutrofia como diagnóstico mais frequente em CTIs quando considerado o IMC $(53,7 \%$ e $36,4 \%$ da amostra, respectivamente). Quando avaliados os desvios nutricionais, apenas $9,7 \%$ e $4,8 \%$ dos pacientes, respectivamente, foram classificados como desnutridos nos estudos supracitados, e altas taxas de sobrepeso e obesidade foram encontradas (SANTOS; VIANA, 2016; ROBINSON et al., 2015).

Quanto à CB, o valor encontrado foi superior ao observado em outros estudos, como de Martins et al. (2017), no qual a classificação de desnutrição de qualquer grau foi identificada na maioria dos pacientes internados em CTI de hospital filantrópico em Minas Gerais (38,2\%), porém ainda representou parcela menor do que a identificada no CTI em questão.

Apesar da alta prevalência de desnutrição, os demais dados encontrados associados à literatura atual permitem sugerir a presença de transição 
nutricional nas unidades de terapia intensiva, já que não se predomina somente a desnutrição, mas também outro padrão nutricional, representado pelo excesso de peso (MARTINS et al., 2017).

Ademais, sabe-se que a avaliação nutricional de pacientes críticos é complexa, especialmente pelas condições clínicas desse grupo, que podem incluir fatores que dificultem sua realização, como a presença de edema, imobilidade, entre outros. Ainda assim, o uso do IMC, calculado por meio do peso e da altura estimados, é uma importante ferramenta utilizada na prática clínica e que parece ter relação com o prognóstico dos pacientes, como proposto por Freitas, Nogueira e Hessel (2015), que verificaram que pacientes críticos recebendo NP com menores valores de IMC apresentaram maiores taxas de mortalidade. Dessa forma, a majoritariedade de indivíduos eutróficos encontrada no presente estudo poderia ser considerada positiva. Contudo é necessário considerar que a alta prevalência de desnutrição identificada tanto pelo IMC quanto pela CB pode ter contribuído para os elevados índices de mortalidade detectados na amostra em questão.

O tempo médio de uso de NP (13,8 \pm 10,5 dias) foi similar ao encontrado por estudo realizado com pacientes de todos os setores de um hospital terciário, que totalizou 11,6 $\pm 6,48$ dias (AMARAL et al., 2015). A terapia nutricional mais utilizada foi a associação entre nutrição enteral e parenteral, ressaltando o elevado uso do suporte enteral como dieta de trânsito (93,3\% dos casos de associação). Tal achado é positivo, tendo em vista que o uso de nutrição enteral propicia a distribuição de nutrientes no trato gastrointestinal que, mesmo em pequena quantidade, podem colaborar para recuperação nutricional e a manutenção do trofismo do epitélio intestinal (SANTOS et al., 2015).

A análise da oferta média de calorias e macronutrientes demonstrou que o valor calórico, glicídico e proteico recebido pelos pacientes estava adequado segundo o preconizado pela Espen. Apenas o aporte lipídico apresentou-se inferior ao recomendado, com 85,56 \pm 18,96\% de adequação entre os pacientes. De forma semelhante, Freitas, Nogueira e Hessel (2015) encontraram valores adequados caloricamente e em termos de macronutrientes em pacientes críticos recebendo NP em um hospital terciário em Campinas, SP. Amaral et al. (2015) também avaliaram as necessidades 
nutricionais de pacientes em uso de NP e obtiveram adequação de 95,5 $\pm 24 \%$ para o aporte energético, $104,3 \pm 26,1 \%$ para as proteínas, $101,3 \pm$ $27,4 \%$ para carboidratos e $96,8 \pm 40,6 \%$ para os lipídios, corroborando os achados sobre adequação da NP nesse grupo.

Apesar dos resultados encontrados serem considerados positivos, destaca-se que as médias obtidas e o cálculo da adequação não isentam a presença de discrepância entre os valores recomendados e prescritos para calorias e macronutrientes. Essa relação está descrita em outros trabalhos em que, apesar do percentual de adequação mostrar-se apropriado, o valor calórico foi inferior ao determinado em $65 \%$ dos indivíduos, e houve superestimação da necessidade proteica, lipídica e de carboidratos em 65\%, $40 \%$ e $45 \%$ dos pacientes, respectivamente (AMARAL et al., 2015). Embora em menores proporções, registrou-se no presente estudo aporte calórico excessivo em 19,0\% da população, deficit proteico em 19,0\% e superestimação da necessidade de proteínas em 38,0\%, além de aporte lipídico inferior ao recomendado em $52,6 \%$ dos casos.

Sob esse contexto, destaca-se o aporte proteico insuficiente identificado especificamente em dois casos em que os pacientes receberam NP plena. Ambos foram classificados como obesos segundo o IMC, sendo provável que o estado nutricional não tenha sido levado em consideração para o planejamento nutricional. Apesar de a Espen não orientar quanto aos desvios nutricionais, as diretrizes da Aspen propõem a terapia nutricional em pacientes críticos obesos, para os quais a dieta deve ser hiperproteica, com recomendação de $2,0 \mathrm{~g} / \mathrm{kg}$ de peso ideal/dia para aqueles com IMC entre 30 $\mathrm{kg} / \mathrm{m}^{2}$ e $40 \mathrm{~kg} / \mathrm{m}^{2}$, como era o caso dos pacientes descritos (MCCLAVE et al., 2016). Dessa forma, ressalta-se a importância de avaliação nutricional e planejamento adequados durante o tratamento dos pacientes críticos.

A ocorrência de instabilidade hemodinâmica neste estudo foi de $47,6 \%$, e houve elevada proporção de hiperglicemia $(45,0 \%)$ e associação de episódios de hipo e hiperglicemia (25,0\%). Distúrbios hemodinâmicos e glicêmicos frequentemente são reportados em estudos sobre o uso de NP, a exemplo do observado por López et al. (2018), em pacientes críticos em Ourense, na Espanha; em tal pesquisa, identificou-se hiperglicemia em $98 \%$ dos pacientes e $16 \%$ apresentaram hipoglicemia. Considerando que 
esses parâmetros podem impactar em desconforto ao paciente e aumento na morbimortalidade (GOMES; FOSS; FOSS-FREITAS, 2014), salienta-se a relevância de monitorização dos pacientes críticos, a fim de possibilitar sua correção e a otimização terapêutica, de forma geral.

Observou-se que a maior parte dos pacientes $(76,2 \%)$ recebeu NP plena durante a internação, contudo 40,0\% das mulheres não atingiram o aporte planejado. O motivo para tal ocorrência não foi alvo de investigação deste estudo, porém podemos elencar como prováveis causas os eventos adversos, a gravidade dos casos clínicos e a possibilidade de o óbito ocorrer antes do alcance de NP plena. Assim, faz-se necessário maior investigação acerca dos fatores que impedem o recebimento total do aporte planejado, a fim de estabelecer medidas de intervenção para melhora nesse quadro.

\section{CONCLUSÃO}

Conclui-se que a maioria dos pacientes críticos internados no CTI é do sexo masculino, e há alta prevalência de idosos nessa população. Apesar de majoritariamente apresentarem IMC indicativo de eutrofia, o baixo peso também foi observado em grande parte da amostra, evidenciando deficit nutricional significativo no grupo avaliado, em especial quando considerada a CB.

O presente estudo permitiu identificar alta incidência de mortalidade, longo tempo de permanência no CTI e elevada ocorrência de distúrbios glicêmicos e instabilidade hemodinâmica em pacientes críticos.

Além disso, apesar do aporte calórico, de carboidratos e proteínas apresentar-se adequado de um modo geral, é preciso considerar as inconformidades detectadas nas prescrições de alguns pacientes, a exemplo daqueles com excesso de peso.

Considerando que a oferta nutricional insuficiente ou excessiva no ambiente de terapia intensiva pode contribuir para a ocorrência de eventos adversos, declínio no quadro clínico e até mesmo acarretar óbito, destaca-se a importância da realização de avaliação nutricional completa nesses pacientes para estabelecer seu diagnóstico nutricional e auxiliar no planejamento adequado da terapia nutricional. Ainda, são fundamentais 
o monitoramento e a reavaliação constantes dos pacientes e da conduta nutricional adotada.

Diante do exposto, deve ser considerada a implementação de estratégias como o desenvolvimento de protocolos para padronização das prescrições e dos métodos que permitam monitoração da nutrição artificial, visando contribuir para a recuperação do estado nutricional e clínico dos pacientes internados em CTI.

\section{REFERÊNCIAS}

ALBUQUeRQUE, J. M.; SILVA, R. F. A.; SOUZA, R. F. F. Perfil epidemiológico e seguimento após alta de pacientes internados em unidade de terapia intensiva. Cogitare Enfermagem, Curitiba, v. 22, n. 3, 2017.

AMARAL, E. B.; BUHLER, F. V.; GONÇALVES, C. B. C.; SOUZA, A. P. Avaliação das prescrições de nutrição parenteral de pacientes adultos internados em hospital terciário. Revista Brasileira de Nutrição Clínica, Porto Alegre, v. 30, n. 2, p. 106-10, 2015.

AMERICAN DIABETES ASSOCIATION (ADA). Standards of Medical Care in Diabetes. The Journal of Clinical and Applied Research and Education: Diabetes Care, v. 40, n. 1, p. 1-135, jan. 2017.

BLACKBURN, G. L.; THORNTON, P. A. Nutritional assessment of the hospitalized patient. Medical Clinics of North America, v. 63, n. 5, p. 1103-15, set. 1979.

EL-FAKHOURI, S.; CARRASCO, H. V. C. G.; ARAÚJO, G. C.; FRINI, I. C. M. Perfil epidemiológico dos pacientes da UTI da Faculdade de Medicina de Marília. Revista da Associação Médica Brasileira, São Paulo, v. 62, n. 3, p. 248-54, 2016.

FREITAS, R. G. B. O. N.; NOGUEIRA, R. J. N.; HESSEL, G. Protein needs of critically ill patients receiving parenteral nutrition. Nutrición Hospitalaria, Madrid, v. 32, n. 1, p. 250-5, 2015.

GOMES, P. M.; FOSS, M. G.; FOSS-FREITAS, M. C. Controle da hiperglicemia intrahospitalar em pacientes críticos e não-críticos. Revista Medicina (Ribeirão Preto), v. 47, n. 2, p. 194-200, 2014.

LIPSCHITZ, D. A. Screening for nutritional status in the elderly. Primary Care, v. 21, n. 1, p. 55-67, 1994. 
LÓPEZ, A.; VARELA, J. J.; CID, M. M.; COUÑAGO, M.; GAGO, N. Complicaciones hidroelectrolíticas e infecciosas en un año de nutrición parenteral en cuidados críticos. Revista Española de Anestesiología y Reanimación, v. 65, n. 7, p. 373-9, 2018.

MARTINS, R. C. F. C.; VITAL, W. C.; AMARAL, J. F.; VOLP, A. C. P. Perfil nutricional de pacientes internados em unidade de terapia intensiva. Nutrición Clínica y Dietética Hospitalaria, v. 37, n. 4, p. 40-7, 2017.

MCCLAVE, S. A.; TAYLOR, B. E.; MARTINDALE, R. G.; WARREN, M. M.; JOHNSON, D. R.; BRAUNSCHWEIG, C.; MCCARTHY, M. S.; DAVANOS, E.; RICE, T. W.; CRESCI, G. A.; GERVASIO, J. M.; SACKS, G. S.; ROBERTS, P. R.; COMPHER, C. Guidelines for the provision and assessment of nutrition support therapy in the adult critically ill patient: Society of Critical Care Medicine and American Society for Parenteral and Enteral Nutrition. Journal of Parenteral and Enteral Nutrition, v. 40, n. 2, p. 159-211, fev. 2016.

ORGANIZAÇÃO MUNDIAL DA SAÚDE (OMS). Physical status: the use and interpretation of anthropometry. Genebra: World Health Organization, 1995.

ROBINSON, M. K.; MOGENSEN, K. M.; CASSEY, J. D.; MCKANE, C. K. L.; MOROMIZATO, T.; RAWN, J. D.; CHRISTOPHER, K. B. The relationship among obesity, nutrition status, and mortality in the critically ill. Critical Care Medicine, v. 43, n. 1, p. 87100, jan. 2015.

RODRIGUEZ, A. H.; BUB, M. B. C.; PERÃO, O. F.; ZANDONADI, G.; RODRIGUEZ, M. J. H. Características epidemiológicas e causas de óbitos em pacientes internados em terapia intensiva. Revista Brasileira de Enfermagem, Brasília, v. 69, n. 2, p. 229-34, mar./abr. 2016.

ROQUE, K. E.; TONINI, T.; MELO, E. C. P. Eventos adversos na unidade de terapia intensiva: impacto na mortalidade e no tempo de internação em um estudo prospectivo. Cadernos de Saúde Pública, Rio de Janeiro, v. 32, n. 10, p. 1-15, out. 2016.

SANTOS, F. A.; VIANA, K. D. A. L. Avaliação do estado nutricional e da terapêutica dietética de pacientes internados em uma unidade de terapia intensiva. Revista de Pesquisa em Saúde, São Luís, MA, v. 17, n. 1, p. 42-6, jan./abr., 2016.

SANTOS, L. A. A.; DORNA, M. S.; VULCANO, S. B.; AUGUSTI, L.; FRANZONI, L. C.; GONDO, F. F. Terapia nutricional nas doenças inflamatórias intestinais: artigo de revisão. Revista Nutrire, São Paulo, v. 40, n. 3, p. 383-96, dez. 2015. 
SINGER, P.; BERGER, M. M.; BERGHE, G. V. den; BIOLO, G.; CALDER, P.; FORBES, P. A.; GRIFFITHS, R.; KREYMAN, G.; LEVERVE, X.; PICHARD, C. ESPEN Guidelines on parenteral nutrition: intensive care. Clinical Nutrition, v. 28, p. 387-400, 2009. 
